Draft Version November 29, 2018

Preprint typeset using $\mathrm{LT}_{\mathrm{E}} \mathrm{X}$ style emulateapj v. 6/22/04

\title{
TURBULENT DYNAMOS WITH SHEAR AND FRACTIONAL HELICITY
}

Petri J. KäPylä ${ }^{1}$ And Axel Brandenburg ${ }^{2}$

Draft version November 29, 2018

\begin{abstract}
Dynamo action owing to helically forced turbulence and large-scale shear is studied using direct numerical simulations. The resulting magnetic field displays propagating wave-like behavior. This behavior can be modelled in terms of an $\alpha \Omega$ dynamo. In most cases super-equipartition fields are generated. By varying the fraction of helicity of the turbulence the regeneration of poloidal fields via the helicity effect (corresponding to the $\alpha$-effect) is regulated. The saturation level of the magnetic field in the numerical models is consistent with a linear dependence on the ratio of the fractional helicities of the small and large-scale fields, as predicted by a simple nonlinear mean-field model. As the magnetic Reynolds number $\left(\operatorname{Re}_{M}\right)$ based on the wavenumber of the energy-carrying eddies is increased from 1 to 180, the cycle frequency of the large-scale field is found to decrease by a factor of about 6 in cases where the turbulence is fully helical. This is interpreted in terms of the turbulent magnetic diffusivity, which is found to be only weakly dependent on $\operatorname{Re}_{M}$.
\end{abstract}

Subject headings: MHD - turbulence

\section{INTRODUCTION}

Several classes of turbulent astrophysical bodies including stars with outer convection zones and spiral galaxies can contain pronounced large-scale magnetic fields. The scales of these fields are much larger than the scale of the energycontaining eddies of the turbulence responsible for producing these fields. Explaining such fields is an important aim of dynamo theory. Today, turbulence simulations begin to reproduce the phenomenon of large-scale field generation (e.g. Brandenburg 2001; Brandenburg et al. 2001, 2008a; Yousef et al. 2008a,b; Käpylä et al. 2008, 2009a; Hughes \& Proctor 2009) and allow making contact with mean-field dynamo theory, which parameterizes the effects of small-scale correlations such as kinetic helicity on the evolution of the largescale field (Moffatt 1978; Parker 1979; Krause \& Rädler 1980). Indeed, helicity has long been known to facilitate the production of large-scale fields under the condition that an appropriately defined dynamo number exceeds a critical value.

Significant progress in the nonlinear formulation of largescale dynamo theory has been possible by carefully designing and comparing simulations of turbulent magnetic fields with mean-field theory. It is important that both are applied to equivalent situations that are sufficiently simple (simple geometries and boundary conditions, homogeneity of the turbulence, etc). Particularly illuminating is the case with triplyperiodic boundary conditions where the energy of the largescale field can exceed the energy of the small-scale field by a factor that is equal to the scale separation ratio, i.e. the scale of the system divided by the scale of the energy-carrying eddies. The phenomenology of this behavior based on magnetic helicity conservation was already described by Brandenburg (2001). He found that full saturation occurs on a resistive time scale following a characteristic einschalt or switch-on curve pattern. The corresponding nonlinear mean-field theory for helical dynamos was developed by Field \& Blackman (2002), and extended to the case with shear by Blackman \& Brandenburg (2002, hereafter BB02).

\footnotetext{
1 Observatory, University of Helsinki, PO Box 14, FI-00014 University of Helsinki, Finland

2 NORDITA, Roslagstullsbacken 23, SE-10691 Stockholm, Sweden Revision: 1.101 (November 29, 2018)
}

One of the important and surprising findings since the early works of Cattaneo \& Vainshtein (1991), Vainshtein \& Cattaneo (1992), Gruzinov \& Diamond (1994), and Bhattacharjee \& Yuan (1995) was the realization that the value of the magnetic Reynolds number, $\operatorname{Re}_{M}$, enters the nonlinear meanfield theory. Even today simulations have not yet been able to establish rigorously that the large-scale dynamo solutions obtained so far are asymptotically independent of the value of $\operatorname{Re}_{M}$. There are many aspects of this problem; the most important one is probably the possibility of catastrophic $\alpha$ quenching. This means that the field cannot saturate to equipartition strength on a dynamical timescale and that in a closed or periodic domain fields of equipartition strength can only be reached on a resistive timescale. It is fairly clear now that catastrophic quenching can only be alleviated in the presence of magnetic helicity fluxes (Blackman \& Field 2000; Kleeorin et al. 2000). What is less clear, however, is whether the helicity fluxes themselves depend on $\operatorname{Re}_{M}$ and on the mean field.

Numerical evidence for large-scale fields in the presence of boundaries comes from simulations of both forced turbulence (Brandenburg 2005) as well as convection (Käpylä et al. 2008). The latter reference was particularly effective in explaining the reason for the absence of a significant large-scale field in the simulations of Tobias et al. (2008), even though their boundary conditions would have allowed a helicity flux. The reason is that the helicity flux follows the direction of the contours of constant shear (Brandenburg \& Subramanian 2005b), but in the simulations of Tobias et al. (2008) these contours do not cross an open surface, because they used periodic boundary conditions in the lateral direction. When using instead open boundary conditions in the lateral direction, a strong large scale field is obtained (see Figure 17 in Käpylä et al. 2008). Alternatively, one can use vertical contours of constant shear, as was done in Käpylä et al. (2008). This was recently confirmed by Hughes \& Proctor (2009) in an independent study.

In the absence of magnetic helicity fluxes and without boundaries, strong large-scale fields can only be generated on a resistive timescale (Brandenburg 2001; Brandenburg et al. 2001). With boundaries, and in the absence of magnetic helicity fluxes, the strength of large-scale magnetic fields decreases 
like $\mathrm{Re}_{M}^{-1 / 2}$ with increasing values of $\mathrm{Re}_{M}$; see Brandenburg \& Subramanian (2005b). Recent simulations of rigidly rotating convection have also been successful in generating a large-scale dynamo (Käpylä et al. 2009a). There, however, the $\mathrm{Re}_{M}$-coverage is not sufficient to determine a scaling for the saturation level. In the following we focus on the former case using periodic boundary conditions, so no magnetic helicity can leave the domain, but large-scale fields can still emerge on a resistive timescale. We use such a configuration to study the quenching of the turbulent magnetic diffusivity, $\eta_{\mathrm{t}}$, and whether its value depends on $\operatorname{Re}_{M}$. The quenched value of $\eta_{\mathrm{t}}$ is crucial for determining the cycle period in an oscillatory $\alpha \Omega$ dynamo. Indeed, BB 02 showed that in the saturated regime and under the assumption of homogeneity the cycle frequency $\omega_{\text {cyc }}$ of an $\alpha \Omega$ dynamo can be written as

$$
\omega_{\text {cyc }}=\eta_{\mathrm{T}} k_{\mathrm{m}}^{2}
$$

where $\eta_{\mathrm{T}}=\eta_{\mathrm{t}}+\eta$ is the total (turbulent plus microscopic) magnetic diffusivity in the quenched state and $k_{\mathrm{m}}$ is the wavenumber of the mean magnetic field. Experiments with nonlinear mean-field models suggest that Equation (1) remains approximately valid even in the mildly nonlinear regime.

Equation (1) applies to homogeneous dynamo waves of sufficiently low amplitude, so that the field variation remains harmonic in space and time. Such behavior can only be modeled with periodic boundary conditions in the direction perpendicular to the plane of the shear flow. The result is expected to change in the presence of boundary conditions, but $\omega_{\text {cyc }}$ should still be proportional to $\eta_{\mathrm{T}}$. Therefore, any $\mathrm{Re}_{M^{-}}$ dependent quenching of $\eta_{\mathrm{T}}$ would directly affect $\omega_{\text {cyc }}$, regardless of whether or not magnetic helicity fluxes would alleviate catastrophic $\alpha$ quenching. This underlines the importance of studying the quenching of $\eta_{\mathrm{t}}$.

Determining the quenching of $\eta_{\mathrm{t}}$ has been attempted on various occasions in the past. It is now generally accepted that, in two dimensions, $\eta_{\mathrm{t}}$ is catastrophically quenched proportional to $1 / \operatorname{Re}_{M}$, provided the energy of the mean field is comparable to the energy of the turbulence. However, this is because in two dimensions the mean squared magnetic vector potential is conserved, which would not be applicable in three dimensions (Gruzinov \& Diamond 1995). For three-dimensional turbulence, analytic theory predicts $\eta_{\mathrm{t}}$ quenching proportional to the strength of the mean field independently of $\operatorname{Re}_{M}$. Such a behavior also emerges from forced turbulence simulations where an initially sinusoidal magnetic field is found to decay proportional to $\exp \left(-\eta_{\mathrm{T}} k_{\mathrm{m}}^{2} t\right)$ (Yousef et al. 2003). The inferred quenching formula, applied to the corresponding mean-field dynamo models, is also found to produce the best fit to simulations that do show strong large-scale dynamo action (BB02). More recently, Brandenburg et al. (2008b) have computed the full $\alpha_{i j}$ and $\eta_{i j}$ tensors in the saturated state without shear, where the mean field is a Beltrami field. In that case, the turbulent magnetic diffusivity was found to be reduced by a factor of about 5 as $\operatorname{Re}_{M}$ is increased from 2 to 600 . However, the case of Beltrami fields has certain limitations that are avoided when there is shear.

\section{THE SIMULATIONS}

We solve the stochastically forced isothermal hydromagnetic equations in a cubical domain of size $(2 \pi)^{3}$ in the presence of a uniform shear flow, $\boldsymbol{U}_{0}=(0, S x, 0)$, with $S=$ const,

$$
\frac{\mathcal{D} \boldsymbol{A}}{\mathcal{D} t}=-S A_{y} \hat{\boldsymbol{x}}-(\boldsymbol{\nabla} \boldsymbol{U})^{T} \boldsymbol{A}+\eta \nabla^{2} \boldsymbol{A},
$$

$$
\begin{gathered}
\frac{\mathcal{D} \boldsymbol{U}}{\mathcal{D} t}=-S U_{x} \hat{\boldsymbol{y}}-c_{\mathrm{s}}^{2} \boldsymbol{\nabla} \ln \rho+\frac{1}{\rho} \boldsymbol{J} \times \boldsymbol{B}+\boldsymbol{F}_{\mathrm{visc}}+\boldsymbol{f}, \\
\frac{\mathcal{D} \ln \rho}{\mathcal{D} t}=-\boldsymbol{\nabla} \cdot \boldsymbol{U},
\end{gathered}
$$

where $\mathcal{D} / \mathcal{D} t=\partial / \partial t+\left(\boldsymbol{U}+\boldsymbol{U}_{0}\right) \cdot \boldsymbol{\nabla}$ is the advective derivative with respect to the total flow velocity that also includes the shear flow, and $\left[(\boldsymbol{\nabla} \boldsymbol{U})^{T} \boldsymbol{A}\right]_{i}=U_{j} A_{i, j}$ in component form, $\boldsymbol{F}_{\text {visc }}$ is the viscous force, and $f$ is the forcing term. As in earlier work (Brandenburg 2001), the forcing function is given by

$$
\boldsymbol{f}(\boldsymbol{x}, t)=\operatorname{Re}\left\{N \boldsymbol{f}_{\boldsymbol{k}(t)} \exp [\mathrm{i} \boldsymbol{k}(t) \cdot \boldsymbol{x}+\mathrm{i} \phi(t)]\right\},
$$

where $\boldsymbol{x}$ is the position vector. The wavevector $\boldsymbol{k}(t)$ and the random phase $-\pi<\phi(t) \leq \pi$ change at every time step, so $\boldsymbol{f}(\boldsymbol{x}, t)$ is $\delta$-correlated in time. For the time-integrated forcing function to be independent of the length of the time step $\delta t$, the normalization factor $N$ has to be proportional to $\delta t^{-1 / 2}$. On dimensional grounds it is chosen to be $N=f_{0} c_{\mathrm{s}}\left(|\boldsymbol{k}| c_{\mathrm{S}} / \delta t\right)^{1 / 2}$, where $f_{0}$ is a nondimensional forcing amplitude. At each timestep we select randomly one of many possible wavevectors in a certain range around a given forcing wavenumber. The average wavenumber is referred to as $k_{\mathrm{f}}$. We force the system with transverse helical waves,

$$
\boldsymbol{f}_{\boldsymbol{k}}=\mathrm{R} \cdot \boldsymbol{f}_{\boldsymbol{k}}^{(\mathrm{nohel})} \quad \text { with } \quad \mathrm{R}_{i j}=\frac{\delta_{i j}-\mathrm{i} \sigma \epsilon_{i j k} \hat{k}_{k}}{\sqrt{1+\sigma^{2}}},
$$

where $\sigma=1$ for the fully helical case with positive helicity of the forcing function,

$$
\boldsymbol{f}_{\boldsymbol{k}}^{(\text {nohel })}=(\boldsymbol{k} \times \hat{\boldsymbol{e}}) / \sqrt{\boldsymbol{k}^{2}-(\boldsymbol{k} \cdot \hat{\boldsymbol{e}})^{2}},
$$

is a non-helical forcing function, and $\hat{\boldsymbol{e}}$ is an arbitrary unit vector not aligned with $\boldsymbol{k}$; note that $\left|\boldsymbol{f}_{\boldsymbol{k}}\right|^{2}=1$.

We use periodic boundary conditions in the $y$ - and $z$ directions and shearing-periodic boundary conditions in the $x$-direction. The main control parameters in our simulations are the magnetic Reynolds and Prandtl numbers, as well as the shear parameter,

$$
\operatorname{Re}_{M}=\frac{u_{\mathrm{rms}}}{\eta k_{\mathrm{f}}}, \quad \operatorname{Pr}_{M}=\frac{v}{\eta}, \quad \mathrm{Sh}=\frac{S}{u_{\mathrm{rms}} k_{\mathrm{f}}} .
$$

By setting $k_{1}=c_{\mathrm{s}}=\rho_{0}=\mu_{0}=1$, we obtain dimensionless units of length, velocity, density, and magnetic field as

$$
[x]=k_{1}^{-1}, \quad[u]=c_{\mathrm{s}}, \quad[\rho]=\rho_{0}, \quad[B]=\sqrt{\mu_{0} \rho_{0}} c_{\mathrm{s}} .
$$

We solve the governing equations using the PencIL $\mathrm{CoDE}^{3}$ which is a high-order finite-difference code (sixth order in space and third order in time) for solving partial differential equations on massively parallel machines.

The three essential parameters varied in the present study are the root-mean-square (rms) velocity (by changing the forcing amplitude $f_{0}$ ), the relative helicity of the turbulence (by changing $\sigma$ ) and the microscopic magnetic diffusivity $\eta$. The rms velocity varies between $0.03-0.2$, corresponding to $f_{0}=0.01-0.05$. The large-scale shear varies between $S=-0.05, \ldots,-0.2$ and leads to shear parameters $\mathrm{Sh} \approx-0.1, \ldots,-1.4$. The high Sh cases studied in Brandenburg \& Käpylä (2007) tend to produce very strong magnetic fields. The purpose of the study is to investigate the saturated state with different values of the mean magnetic field, $\bar{B} / B_{\text {eq }}$, so we need to regulate its value. One way to decrease the

\footnotetext{
${ }^{3}$ http://www.nordita.org/software/pencil-code
} 

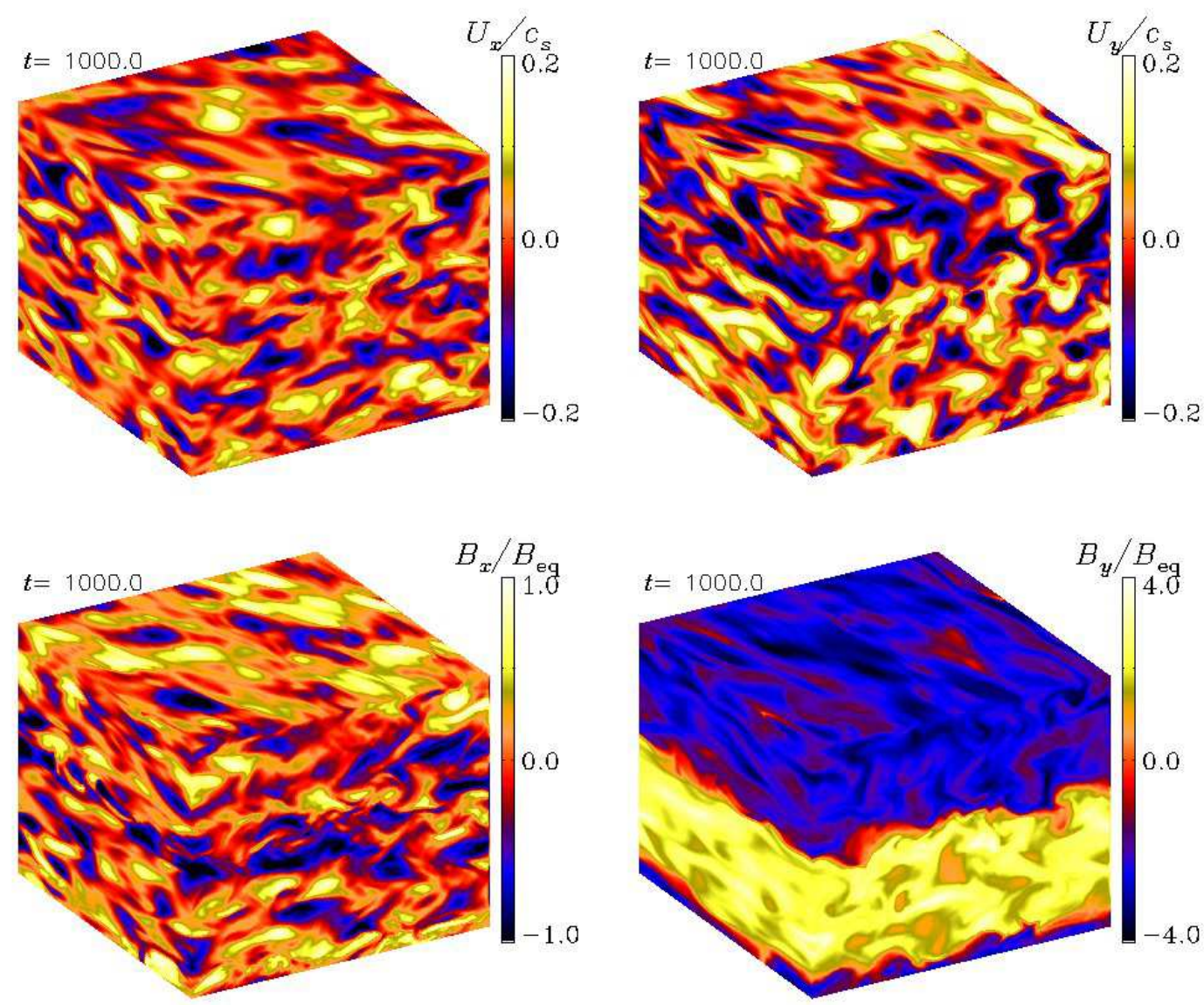

FIG. 1.- $U_{\mathrm{x}}, U_{\mathrm{y}}, B_{\mathrm{x}}$, and $B_{\mathrm{y}}$ at the periphery of the domain from a run with $\operatorname{Re}_{M} \approx 209, \mathrm{Sh} \approx-0.18$, and $\operatorname{Pr}_{M}=10$. Note the absence of any large-scale pattern in $U_{y}$, but a rather pronounced pattern in $B_{y}$ with a fairly abrupt change of sign.

magnetic fields is to increase the forcing amplitude to reduce the relative importance of the $\Omega$ effect. On the other hand, varying the value of $\sigma$ can be used to regulate the $\alpha$ effect with given $\mathrm{Sh}$ and $\mathrm{Re}_{M}$. This procedure was first adopted by Maron \& Blackman (2002) and later by Brandenburg et al. (2002) in order to study the onset of large-scale dynamo action. In most cases $\sigma$ is varied between 0.05 and 1, but small values do not always lead to clear large-scale dynamo action. Performing a large set of runs we have been able to cover a range of $\left(\overline{\boldsymbol{B}} / B_{\mathrm{eq}}\right)^{2}$ from 0.3 to 270 and the magnetic Reynolds number varies between 1.5 and roughly 210 . The numerical resolution of the simulations varies between $64^{3}$ and $256^{3}$ grid points. A summary of the runs is given in Table 1.

\section{FORMALISM}

In this section we briefly review some of the main predictions of the dynamical quenching model as applied to homogeneous shear flows (BB02). In this approach, the quenching of $\alpha$ comes from an additional contribution that is proportional to the small-scale current helicity, which builds up at the same time as the $\alpha$ effect produces large-scale magnetic fields. The sign of the small-scale current helicity is opposite to that of the large-scale current helicity which, in turn, is also equal to the sign of the $\alpha$ effect. The evolution of the smallscale current helicity is calculated from the magnetic helicity equation. Using this equation together with the mean-field induction equation, BB02 derived expressions for the saturation amplitude, the cycle frequency, and the ratio of toroidal to poloidal field amplitudes in closed form. We begin with a more precise definition of $k_{\mathrm{m}}$ in terms of the resulting mean magnetic field via

$$
k_{\mathrm{m}}^{2}=\mu_{0}\langle\overline{\boldsymbol{J}} \cdot \overline{\boldsymbol{B}}\rangle /\langle\overline{\boldsymbol{A}} \cdot \overline{\boldsymbol{B}}\rangle,
$$

where $\mu_{0}$ is the vacuum permeability, overbars denote mean quantities (later we shall specialize to horizontal averages), and angular brackets denote volume averaging. Preliminary results on turbulent diffusivity determined from the cycle frequency were presented in Brandenburg \& Käpylä (2007). In the present study, we explore a much wider range of parameters and seek to understand the quenching behavior of $\eta_{\mathrm{t}}$ as a function of the magnetic field strength.

For future reference we define at this point an analogously defined wavenumber of the fluctuating field via

$$
k_{\mathrm{f}}^{2}=\mu_{0}\langle\boldsymbol{j} \cdot \boldsymbol{b}\rangle /\langle\boldsymbol{a} \cdot \boldsymbol{b}\rangle .
$$

We also define effective wavenumbers of the fluctuating and mean fields via

$$
\begin{gathered}
\epsilon_{\mathrm{f}} k_{\mathrm{f}}=\mu_{0}\langle\boldsymbol{j} \cdot \boldsymbol{b}\rangle /\left\langle\boldsymbol{b}^{2}\right\rangle, \\
\epsilon_{\mathrm{m}} k_{\mathrm{m}}=\mu_{0}\langle\overline{\boldsymbol{J}} \cdot \overline{\boldsymbol{B}}\rangle /\left\langle\overline{\boldsymbol{B}}^{2}\right\rangle,
\end{gathered}
$$

where $\epsilon_{\mathrm{f}}$ and $\epsilon_{\mathrm{m}}$ are the fractional helicities of the fluctuating and mean fields, respectively. We assume these two fractional 
helicities to be positive, but $k_{\mathrm{f}}$ and $k_{\mathrm{m}}$ can have either sign. In the stationary state the total current helicity must vanish for a closed system (Brandenburg 2001), which means that $k_{\mathrm{f}}$ and $k_{\mathrm{m}}$ must have opposite sign. Throughout this work the forcing function has positive helicity, so $k_{\mathrm{f}}>0$, and therefore $k_{\mathrm{m}}<0$.

BB02 compared their results with those of direct simulations of Brandenburg et al. (2001). Theoretically, in the linear regime and at large magnetic Reynolds numbers, $-k_{\mathrm{m}}$ can be as large as $k_{\mathrm{f}} / 2$ (Brandenburg et al. 2002), but in the nonlinear regime, $-k_{\mathrm{m}}$ will decrease until it reaches $k_{1}$ (Brandenburg 2001). Here we consider nonlinear solutions and will therefore assume $-k_{\mathrm{m}}=k_{1}$.

BB02 predicted that the saturation level of the mean magnetic field is given by

$$
\frac{B_{\text {fin }}^{2}}{B_{\text {eq }}^{2}}=\frac{\epsilon_{\mathrm{f}} k_{\mathrm{f}}}{\epsilon_{\mathrm{m}} k_{1}}-\left(1+\mathrm{Re}_{M}^{-1}\right) .
$$

Here, $\epsilon_{\mathrm{f}} k_{\mathrm{f}}$ and $\epsilon_{\mathrm{m}} k_{1}$ are the effective wavenumbers corresponding to the scale of the forcing and the mean field, respectively, and are defined via Equations (12) and (13). The equipartition value of the magnetic field is defined via

$$
B_{\text {eq }}=\left\langle\mu_{0} \rho \boldsymbol{u}^{2}\right\rangle^{1 / 2} \text {. }
$$

According to the calculations of $\mathrm{BB} 02, \epsilon_{\mathrm{m}}$ is directly proportional to the ratio of cycle frequency to shear rate. Furthermore, $\epsilon_{\mathrm{m}}$ is also proportional to the ratio of poloidal to toroidal magnetic field amplitudes. We have therefore multiple checks on the consistency of this simple model.

\section{RESULTS}

\subsection{Saturation level of the magnetic field}

In most cases we find oscillatory solutions with dynamo waves propagating in the positive $z$ direction. This is indeed expected from mean-field theory, according to which the direction of propagating is given by the sign of the product of $\alpha$ effect and shear. Indeed, positive helicity in the forcing should result in a negative $\alpha$ effect which, together with negative shear, predicts a direction of propagation in the positive $z$ direction.

Figure 1 shows the streamwise components of velocity and magnetic field from a run with $\operatorname{Re}_{M} \approx 209$, Sh $\approx-0.18$, and $\operatorname{Pr}_{M}=10$. The velocity field is irregular while the $y$ component of the magnetic field exhibits clear large-scale structure. The $x$-component of the field also has a systematic large-scale component, but it is hard to see in a single snapshot and without horizontal averaging because its amplitude is much lower than that of the $y$-component.

This dynamo wave is well seen in animations showing the $z$ dependence of $B_{y}$ vs. time, but it becomes particularly clear when the field is averaged over the horizontal directions, indicated here by an overbar. An example of $\bar{B}_{x}(z, t)$ and $\bar{B}_{y}(z, t)$ is shown in Fig. 2] Note again the sharp sign changes of $\bar{B}_{y}(z, t)$. One can now see that the locations of these sharp sign changes coincide with the locations where $\bar{B}_{x}(z, t)$ achieves positive or negative extrema. In fact, by comparing the two panels of Fig. 2. one can verify that a positive extremum of $\bar{B}_{x}(z, t)$ leads to a change of sign of $\bar{B}_{y}\left(z, t^{\prime}\right)$ from a positive value at $t^{\prime}<t$ to a negative value at $t^{\prime}>t$, and vice versa. This is explained by the fact that shear is negative, i.e. $S<0$, which turns a positive $\bar{B}_{x}$ into a negative $\bar{B}_{y}$.

The dynamo wave has a typical anharmonic shape (see Fig. 3), just as has been seen before both in mean-field models (Stix 1972) as well as in direct simulations (Brandenburg et al. 2001). Figure 3 shows flat positive or negative plateaus in $\bar{B}_{y}$, during which the $\bar{B}_{x}$ field was weak, but with a clear time derivative: while $\bar{B}_{y}$ is positive, $\partial \bar{B}_{x} / \partial t$ is also positive. A more careful look reveals that the plateaus are not completely flat, but have a negative time derivative when $\bar{B}_{y}$ is positive (and a positive time derivative when $\bar{B}_{y}$ is negative). This relation can be interpreted as being due to a negative $\alpha$ effect in the relation

$$
\frac{\partial \overline{\boldsymbol{B}}}{\partial t}=\boldsymbol{\nabla} \times(\alpha \overline{\boldsymbol{B}})+\ldots
$$

Using the fact that there is a dynamo wave with propagation speed $c$, we have $\overline{\boldsymbol{B}}=\overline{\boldsymbol{B}}(z-c t)$. Assuming constant $\alpha$, this means that $c \bar{B}_{x}^{\prime}=\alpha \bar{B}_{y}^{\prime}$. Since $c$ is positive (compare Fig. 2), the opposite signs of the time derivatives of $\bar{B}_{x}$ and $\bar{B}_{y}$ suggest that $\alpha<0$. This is in agreement with the fact that the kinetic helicity is positive and mean-field theory suggesting that for isotropic turbulence in the high-conductivity limit (Moffatt 1978, Krause \& Rädler 1980) $\alpha$ is a negative multiple of the kinetic helicity.

The oscillations are also discernible in the kinematic regime especially in runs where $\operatorname{Re}_{M}$ is small enough, so that the small-scale dynamo is not excited (see Fig. 4). The cycle frequency in the kinematic regime appears to be roughly constant in the range $\operatorname{Re}_{M}=2 \ldots 10$. In the following, however, we will be concerned with the nonlinear regime where the dynamo has reached saturation. We study the dependence of the value of $\omega_{\text {cyc }}$ on $\operatorname{Re}_{M}$. Using Equation (1) we then calculate $\eta_{\mathrm{T}}$ and hence $\eta_{\mathrm{t}}$. Figure 5 shows that the resulting value of $\eta_{\mathrm{t}}$ has a value roughly $2.5 \eta_{\mathrm{t} 0}$ for $\operatorname{Re}_{M} \gtrsim 4$, where

$$
\eta_{\mathrm{t} 0}=\frac{1}{3} \tau \overline{\boldsymbol{u}^{2}},
$$

and $\tau$ is the correlation time of the turbulence. The subscript zero refers to the kinematic case which is valid when the magnetic field is weak. For small $\operatorname{Re}_{M}$ the correlation time is no longer determined by the turbulence but rather the microscopic diffusivity $\eta$. Thus, in that limit $\eta_{\mathrm{t}}$ is expected to decreases proportionally to $\eta$. The markedly lower value of $\eta_{\mathrm{t}}$ for $\operatorname{Re}_{M} \approx 2$ can be interpreted in terms of this behavior.

For $\operatorname{Re}_{M} \gg 1$ the saturation formula given in Equation (14) predicts that the ratio $B_{\mathrm{fin}}^{2} / B_{\mathrm{eq}}^{2}$ should be linearly proportional to $\epsilon_{\mathrm{f}} k_{\mathrm{f}} / \epsilon_{\mathrm{m}} k_{1}-\left(1+\eta / \eta_{\mathrm{t} 0}\right)$. This is indeed in reasonable agreement with the numerical data; see Figure 6.

BB02 gave two independent formulae for $\epsilon_{\mathrm{m}}$, one in terms of the resulting ratio of poloidal to toroidal mean fields, $Q^{-1}$, where $Q^{2}=\left\langle\bar{B}_{y}^{2}\right\rangle /\left\langle\bar{B}_{x}^{2}\right\rangle$,

$$
\epsilon_{\mathrm{m}}=\sqrt{2} Q^{-1} \equiv \sqrt{2}\left(\left\langle\bar{B}_{x}^{2}\right\rangle /\left\langle\bar{B}_{y}^{2}\right\rangle\right)^{1 / 2},
$$

and one in terms of the resulting ratio of the cycle frequency to the shear frequency,

$$
\epsilon_{\mathrm{m}}=2 \omega_{\mathrm{cyc}} / S \text {. }
$$

Furthermore, assuming $k_{\mathrm{m}}=-k_{1}$ in Equation (13), we arrive at

$$
\epsilon_{\mathrm{m}}=-\mu_{0} \frac{\langle\overline{\boldsymbol{J}} \cdot \overline{\boldsymbol{B}}\rangle}{k_{1}\left\langle\overline{\boldsymbol{B}}^{2}\right\rangle} .
$$

We use the definition of $\epsilon_{\mathrm{m}}$ given in Equation (20) as the benchmark value to compare the other two expressions to. A scatter plot of the results is given in Figure 7. We find that the values given by Equations (18) and (19) are consistently 
Summary of the DifFerent SETS OF RUNS. Here, $\tilde{B}_{\text {rms }}=B_{\text {rms }} / B_{\text {eq }},\left\langle\tilde{\bar{B}}_{x}^{2}\right\rangle^{1 / 2}=\left\langle\bar{B}_{x}^{2}\right\rangle^{1 / 2} / B_{\text {eq }}$, AND $\left\langle\tilde{\bar{B}}_{y}^{2}\right\rangle^{1 / 2}=\left\langle\bar{B}_{y}^{2}\right\rangle^{1 / 2} / B_{\text {eq }}$.

\begin{tabular}{cccccccccc}
\hline \hline Set & grid & $\operatorname{Re}_{M}$ & $\operatorname{Pr}_{M}$ & $k_{\mathrm{f}} / k_{1}$ & $\mathrm{Sh}$ & $\sigma$ & $\tilde{B}_{\text {rms }}$ & $\left\langle\tilde{\bar{B}}_{x}^{2}\right\rangle^{1 / 2}$ & $\left\langle\tilde{\bar{B}}_{y}^{2}\right\rangle^{1 / 2}$ \\
\hline $\mathrm{A}$ & $64^{3}$ & 1.5 & 1 & 10 & -0.72 & $0.2 \ldots 1$ & $8.0 \ldots 13.0$ & $0.14 \ldots 0.22$ & $7.2 \ldots 11.6$ \\
$\mathrm{~B}$ & $64^{3}$ & $46 \ldots 94$ & 10 & 5 & $-0.33 \ldots-0.16$ & $0.05 \ldots 1$ & $1.5 \ldots 2.4$ & $0.05 \ldots 0.22$ & $0.95 \ldots 1.8$ \\
$\mathrm{C}$ & $64^{3}$ & $27 \ldots 35$ & 10 & 5 & $-0.57 \ldots-0.44$ & $0.05 \ldots 0.7$ & $2.0 \ldots 9.1$ & $0.05 \ldots 0.09$ & $1.5 \ldots 8.2$ \\
$\mathrm{D}$ & $64^{3}$ & $11 \ldots 13$ & 10 & 5 & $-1.43 \ldots-1.16$ & $0.05 \ldots 1$ & $2.7 \ldots 17.9$ & $0.03 \ldots 0.10$ & $1.8 \ldots 16.2$ \\
$\mathrm{E}$ & $128^{3}$ & 11 & 10 & 5 & $-1.42 \ldots-1.36$ & $0.2 \ldots 1$ & $7.9 \ldots 14.4$ & $0.07 \ldots 0.10$ & $7.2 \ldots 13.1$ \\
$\mathrm{~F}$ & $128^{3}$ & $131 \ldots 209$ & 10 & 5 & $-0.29 \ldots-0.18$ & $0.05 \ldots 1$ & $1.2 \ldots 2.9$ & $0.04 \ldots 0.20$ & $0.5 \ldots 2.2$ \\
$\mathrm{G}$ & $256^{3}$ & 29 & 25 & 5 & $-1.38 \ldots-1.29$ & $0.2 \ldots 1$ & $8.3 \ldots 15.9$ & $0.05 \ldots 0.06$ & $7.5 \ldots 14.3$ \\
$\mathrm{H}$ & $64^{3} \ldots 128^{3}$ & $1.4 \ldots 181$ & $0.5 \ldots 50$ & 5 & $-0.13 \ldots-0.10$ & 1 & $1.7 \ldots 3.1$ & $0.23 \ldots 0.55$ & $1.2 \ldots 2.3$ \\
\hline
\end{tabular}

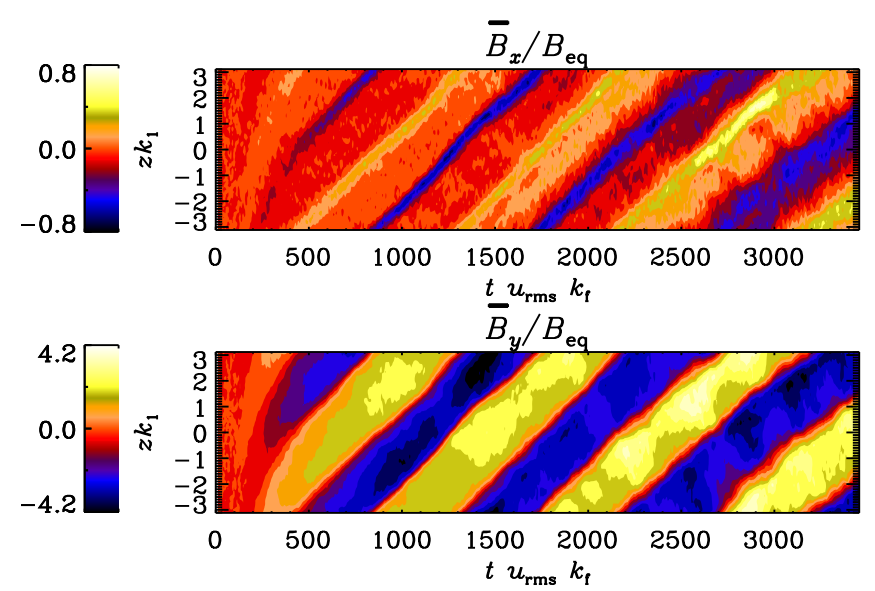

FIG. 2.- Space-time diagrams of $\bar{B}_{x}(z, t)$ (upper panel) and $\bar{B}_{y}(z, t)$ (lower panel). From a run with $\operatorname{Re}_{M} \approx 209, \mathrm{Sh} \approx-0.18$ and $\operatorname{Pr}_{M}=10$.

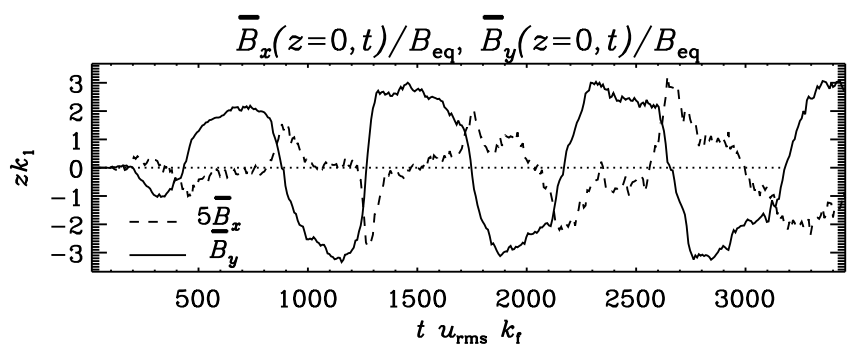

Fig. 3.- Five times $\bar{B}_{x}$ (dashed line) and $\bar{B}_{y}$ (solid line) in the midplane, i.e. $z=0$. From the same run as in Fig. 2

below the value of $\epsilon_{\mathrm{m}}$ as given by Equation (20), but that the trend as a function of $\sigma$ is the same for all three quantities. Thus, we apply scaling factors of the order of unity for the expressions in Eqs. (18) and (19) in Figure 7 so that the data fall onto the same line. An exception are a few points for the cycle frequency, given by Equation (19), where there are departures. The points deviating from the trend all belong to runs that have the weakest mean fields and occur for $\epsilon_{\mathrm{m}}>0.1$.

\subsection{Effects from the vorticity dynamo}

A few runs exhibit signs of intermittent or continuous largescale vorticity generation in the saturated state of the dynamo, reminiscent of a vorticity dynamo (Elperin et al. 2003; Yousef et al. 2008a,b). In Figure 8, we compare the evolution of the rms Mach number of the total (mean and fluctuating) veloc-

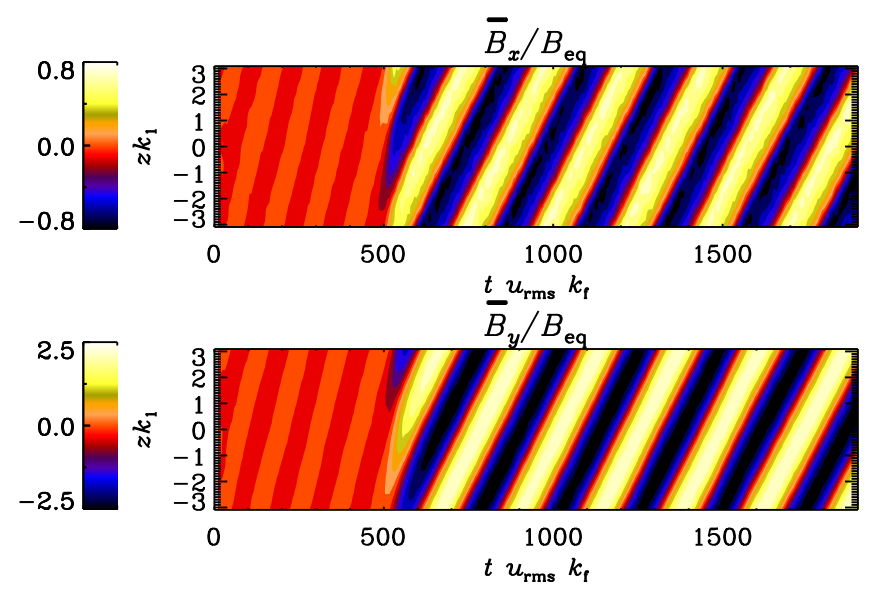

FIG. 4.- Space-time diagrams of $\bar{B}_{x}(z, t)$ (upper panel) and $\bar{B}_{y}(z, t)$ (lower panel) from a run with $\mathrm{Re}_{M} \approx 1.4, \mathrm{Sh} \approx-0.13$ and $\operatorname{Pr}_{M}=1$.

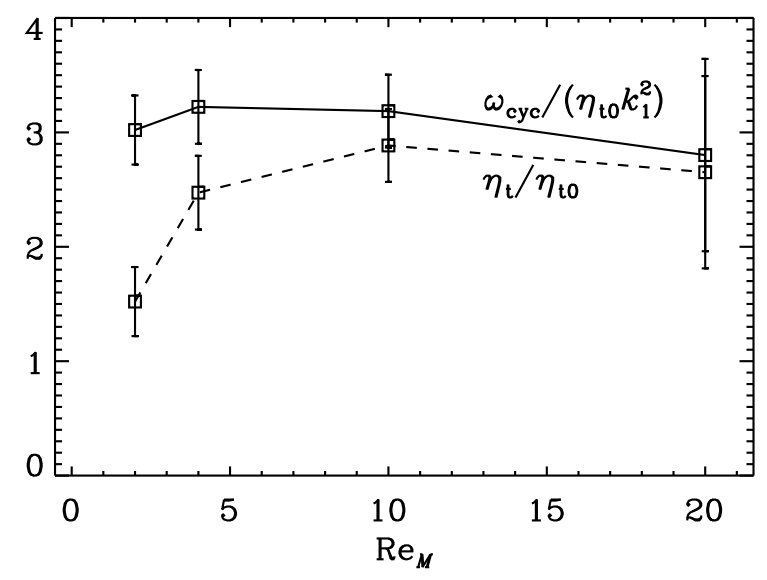

FIG. 5.- Cycle frequency (solid line) and turbulent diffusivity from Equation (1) (dashed line) as functions of $\mathrm{Re}_{M}$ in the kinematic regime.

ity both for hydrodynamic runs with and without shear, as well as a run with magnetic fields and shear. It is clear that in the absence of shear the rms Mach number reaches its final level quite quickly. During the first few tens of turnover times the same level is also maintained in the presence of shear, but the velocity gradually increases to much larger values and reaches a new saturation level that is approximately 


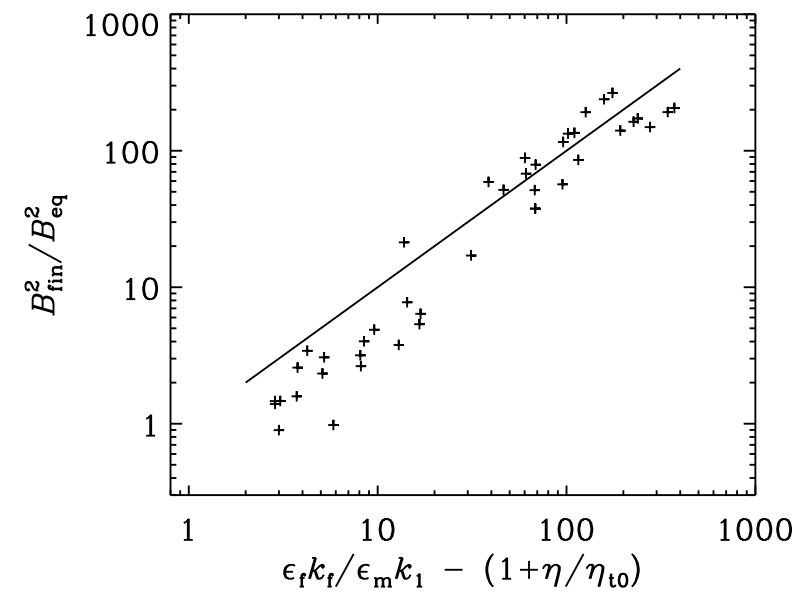

FIG. 6. $-B_{\text {fin }}^{2} / B_{\mathrm{eq}}^{2}$ as a function of $\epsilon_{\mathrm{f}} k_{\mathrm{f}} / \epsilon_{\mathrm{m}} k_{1}-\left(1+\eta / \eta_{\mathrm{t} 0}\right)$. The diagonal is shown for comparison. Note the reasonable agreement with theory (solid line).

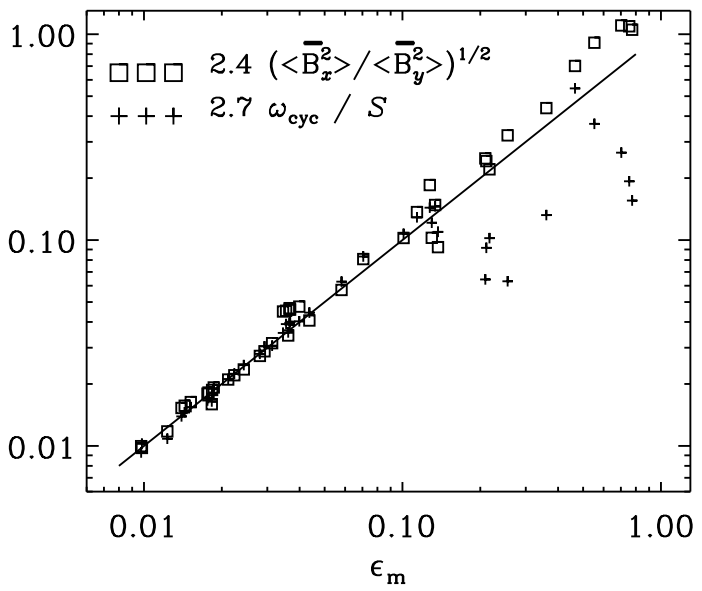

FIG. 7.- Scatter plot of poloidal to toroidal field ratio (squares, scaled by factor 2.7) and normalized cycle frequency (plus signs, scaled by factor 2.4) vs. $\epsilon_{\mathrm{m}}$. Note that for $\epsilon_{\mathrm{m}}<0.1$ both squares and plus signs scatter tightly around the diagonal.

10 times larger. During that time a large-scale velocity field develops throughout the entire domain; see Fig. 9 for a typical example of this behavior. In the hydromagnetic case the vorticity dynamo is quenched when the magnetic field grows to high enough level but in cases of weaker magnetic fields this quenching is often only partial as demonstrated by the dashed line in Figure 8, Unlike the anisotropic kinetic $\alpha$ effect (Frisch et al. 1987), which is also suppressed by magnetic fields (Brandenburg \& von Rekowski 2001), and works only for $\operatorname{Re} \equiv \operatorname{Re}_{M} / \operatorname{Pr}_{M} \leq 8$, the vorticity dynamo has been shown to work well up to $\operatorname{Re} \approx 100$. A more thorough study of the vorticity dynamo can be found elsewhere (Käpylä et al. 2009b).

The overall behavior of such runs is quite different from those where a large-scale flow did not develop. We have therefore excluded these runs from the plots for the sake of clarity. All in all the agreement between the three independent checks of $\epsilon_{\mathrm{m}}$ is remarkably good especially for low values of $\epsilon_{\mathrm{m}}$.
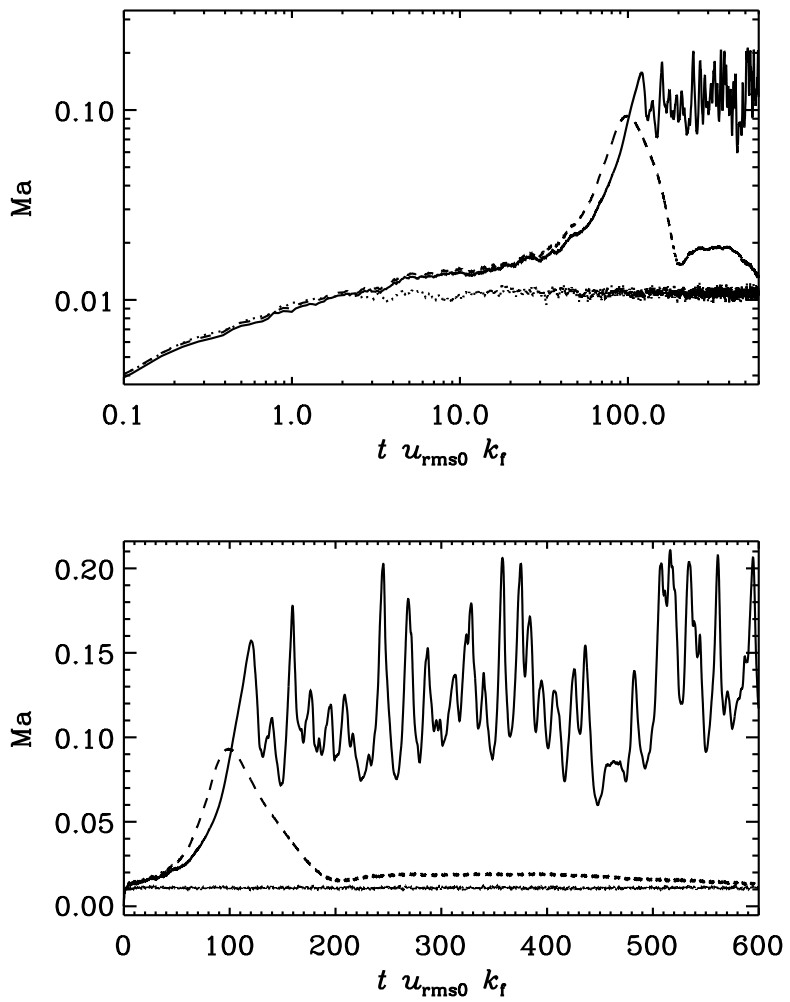

FIG. 8. - Root mean square Mach number for two shearing runs with the same initial parameters $\left(S=-0.2, v=5 \cdot 10^{-3}\right)$ with (dashed line) and without (solid line) magnetic fields. The dotted line shows the result for a non-shearing hydrodynamic run for comparison.

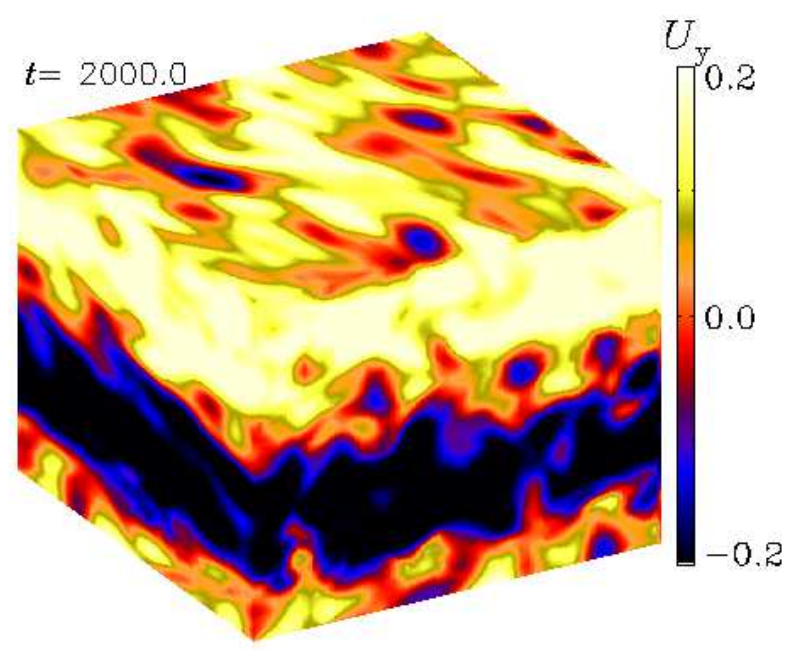

FIG. 9.- $U_{\mathrm{y}}$ at the periphery of the domain from a run with $\mathrm{Re}_{M} \approx 80$, $\mathrm{Sh} \approx-0.19$, and $\operatorname{Pr}_{M}=10$, at a resolution of $64^{3}$ mesh points at a time when the magnetic field is saturated and a large-scale vorticity dynamo develops.

\subsection{Turbulent diffusivity quenching}

According to the first order smoothing approximation (Moffatt 1978, Krause \& Rädler 1980), the turbulent diffusivity for isotropic turbulence in the high-conductivity limit is given by Equation (17). Simulations of forced turbulence 
without shear suggest that, independent of the value of the forcing wavenumber and amplitude, the Strouhal number is around unity (Brandenburg \& Subramanian 2005a, 2007), i.e.

$$
\mathrm{St} \equiv k_{\mathrm{f}} u_{\mathrm{rms}} \tau \approx 1 \text {. }
$$

In the kinematic regime, the magnetic diffusivity can therefore be written as

$$
\eta_{\mathrm{t} 0}=\frac{1}{3} \mathrm{St} u_{\mathrm{rms}} k_{\mathrm{f}}^{-1} \approx \frac{1}{3} u_{\mathrm{rms}} k_{\mathrm{f}}^{-1} .
$$

Recent numerical simulations employing the test-field procedure have shown that in the kinematic case, Equation (22) is confirmed for $\operatorname{Re}_{M}$ between 1 and 200; see Sur et al. (2008). In order to study the quenching of $\eta_{\mathrm{t}}$, i.e. the dependence on $\overline{\boldsymbol{B}}$, we normalize our results with the value of $\eta_{\mathrm{t} 0}$ from a simulation without magnetic fields.

Our setup is similar to that used in a number of related simulations by Brandenburg et al. (2008a) with non-helical turbulence, and Mitra et al. (2009) with helicity. In those simulations the main focus was the determination of turbulent transport coefficients in the linear regime. In these studies it was found that the turbulent diffusivity increases by a factor of a few as $|\mathrm{Sh}|$ increases from 0.1 to unity. More recently, this work has been extended to the nonlinear regime, but so far only in the absence of shear (Brandenburg et al. 2008b). However, there is then a potential difficulty in that there could be additional terms in the functional form of the quenched $\alpha$ and $\eta_{\mathrm{t}}$ tensors that could mimic turbulent diffusion, so the split into two $\alpha$ and $\eta_{\mathrm{t}}$ coefficients is not unique.

In the saturated state the growth rate of the large-scale field is zero. Thus, this situation corresponds to the marginally excited state where the cycle frequency is given by Equation (1). Measuring therefore the dynamo frequency in the saturated state gives the (quenched) value of the turbulent diffusivity.

Results for all of the runs are shown in Figure 10. The data scatter around the curve

$$
\eta_{\mathrm{t}}=\frac{\tilde{\eta}_{\mathrm{t}}}{1+g\left(|\overline{\boldsymbol{B}}| / B_{\mathrm{eq}}\right)^{n}}
$$

with $\tilde{\eta}_{\mathrm{t} 0}=(1.2-1.5) \eta_{\mathrm{t} 0}, g=0.3$ and $n=1-2$ being fit parameters. A factor greater than one in the definition of $\tilde{\eta}_{t 0}$ reflects the fact that in the kinematic regime, the turbulent magnetic diffusivity is enhanced in the presence of shear (Brandenburg et al. 2008a, Mitra et al. 2009). Note that the data for many of the runs with different magnetic Reynolds numbers $\left(\operatorname{Re}_{M}=1 \ldots 210\right)$ seem to fall roughly on the same line. An asymptotic quenching of $\eta_{\mathrm{t}}$ inversely proportional to $\overline{\boldsymbol{B}}$ instead of $\overline{\boldsymbol{B}}^{2}$ has been predicted analytically by Kitchatinov et al. (1994) and Rogachevskii \& Kleeorin (2001), and BB02 found $g \approx 3$ to be a good fit to the numerical simulations of Brandenburg et al. (2001). The fact that $g$ is here different suggests that it is perhaps not a universal constant, but that it may depend on other parameters.

The amount of scatter in Figure 10 does not depend in any systematic way on the value of Sh, except for the lowest values of Sh for which the values of $\eta_{\mathrm{t}}$ fall below the general trend. This, and the fact that $\eta_{\mathrm{t}}$ seems to reach values above unity for low magnetic field strengths, might be a consequence of the turbulent diffusivity being enhanced when shear increases (Brandenburg et al. 2008a, Mitra et al. 2009). In the present case the unquenched value of $u_{\mathrm{rms}}$ from a nonshearing run with the same forcing amplitude has been used in the definition of $\eta_{\mathrm{t} 0}$. If the $u_{\mathrm{rms}}$ from a hydrodynamic run with shear is used, the excitation of the vorticity dynamo would enhance its value by a factor of up to 5 (see Figure 8).

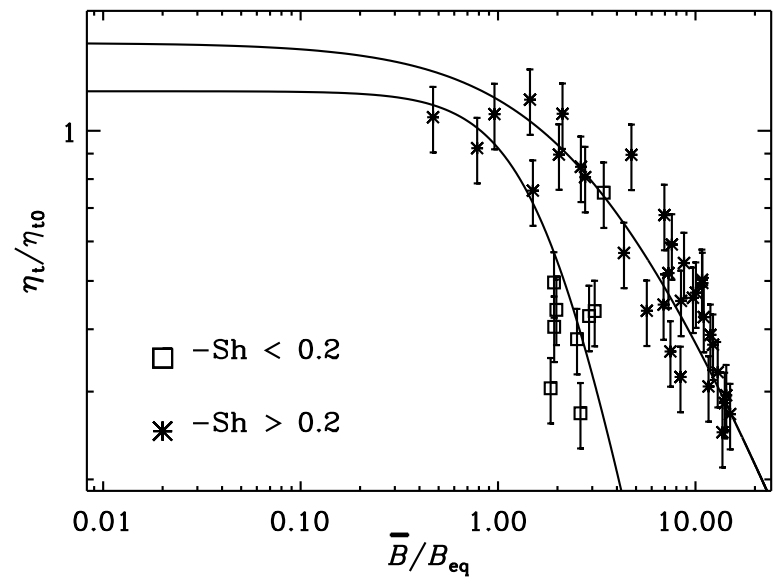

FIG. 10.- Turbulent diffusivity as a function of $|\overline{\boldsymbol{B}}| / B_{\mathrm{eq}}$. The squares and stars denote cases where $-\mathrm{Sh}<0.2$ (weak shear) and $-\mathrm{Sh}>0.2$ (strong shear). The upper curve is for $n=1$ and $\tilde{\eta}_{\mathrm{t} 0} / \eta_{\mathrm{t} 0}=1.5$, while the second curve is for $n=2$ and $\tilde{\eta}_{\mathrm{t} 0} / \eta_{\mathrm{t} 0}=1.2$, and $g=0.3$ in both cases.

In order to study the behavior of $\eta_{\mathrm{t}}$ as a function of $\mathrm{Re}_{M}$ a set of runs were performed where $\operatorname{Pr}_{M}$ was varied between 0.5 and 50, keeping all the other parameters fixed, i.e. $\mathrm{Sh} \approx-0.1$ and $\operatorname{Re}=u_{\mathrm{rms}} /\left(\nu k_{\mathrm{f}}\right) \approx 4$. The results are shown in Figure 11 which demonstrates that $\eta_{\mathrm{t}}$, as inferred from $\omega_{\text {cyc }}$ using Equation (1), decreases by a factor of about 2 when $\mathrm{Re}_{M}$ is increased from 10 to 180 , while $|\overline{\boldsymbol{B}}| / B_{\mathrm{eq}}$ varies from 1.7 to 2.5 , with the larger values occurring at larger values of $\operatorname{Re}_{M}$. The low value of $\eta_{\mathrm{t}}$ for the smallest value of $\mathrm{Re}_{M}$ might derive from the fact that the simple model used to estimate the value of the turbulent diffusivity may not be representative of this regime. On the other hand, there appears to be a declining trend of $\eta_{\mathrm{t}}$ as a function of $\mathrm{Re}_{M}$ approximately proportional to $\operatorname{Re}_{M}^{1 / 3}$. However, higher $\operatorname{Re}_{M}$ simulations are needed to substantiate this. On the other hand, data from nonlinear test-field calculations by Brandenburg et al. (2008b) show that $\eta_{\mathrm{t}}$ decreases by a factor of 5 when $\operatorname{Re}_{M}$ is increased from 2 to 600 . Within error bars this is compatible with the present results.

\section{CONCLUSIONS}

Three dimensional direct numerical simulations of helically forced turbulence with imposed large-scale shear have been used to study the saturation level of the dynamo, its cycle frequency, and thereby the turbulent diffusivity and its quenching with the magnetic field. The parameters of the study were chosen such that a cyclic large-scale magnetic field develops with the dynamo wave propagating to the positive $z$-direction.

We find that the saturation level of the energy of the mean magnetic field is compatible with a scaling of a quantity related to the ratio of the fractional helicities of the small and large-scale fields, in accordance with Equation (14) and the prediction from nonlinear mean-field theory derived by BB02. Furthermore, the three independent measures of $\epsilon_{\mathrm{m}}$ are in reasonably good agreement, especially for small values of $\epsilon_{\mathrm{m}}$.

The turbulent diffusivity is found to be quenched approximately inversely proportional to the large-scale magnetic field strength. This is in agreement with earlier analytical studies (Kitchatinov et al. 1994; Rogachevskii \& Kleeorin 2001). However, a small group of data points can also be fitted to a quadratic dependence on the inverse field strength. The de- 


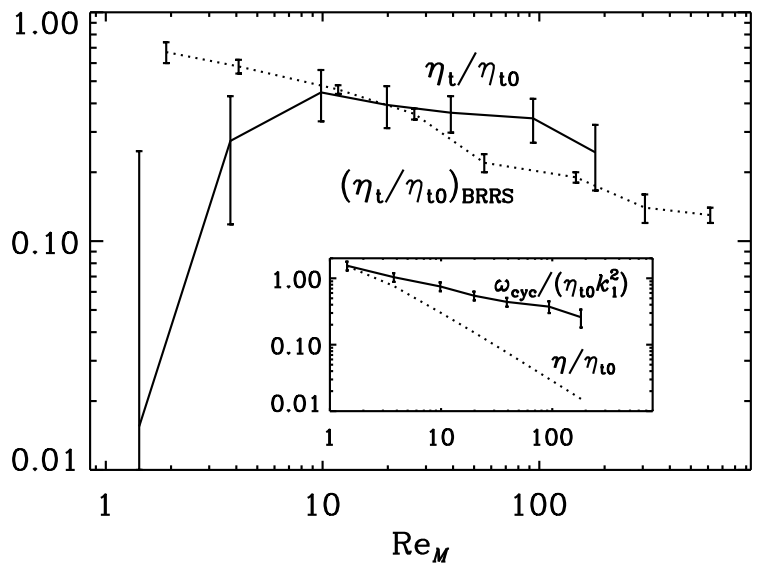

FIG. 11.- Turbulent magnetic diffusivity as a function of $\mathrm{Re}_{M}$, as obtained from the cycle frequency (solid line) for Sh $=-0.1$ and $\sigma=1$, which translates to $\epsilon_{\mathrm{f}} \approx 1$, compared with the corresponding result by Brandenburg et al. (2008b) using the test-field method (Sh $=0)$. The inset shows the $\operatorname{Re}_{M}$ dependence of the cycle frequency $\omega_{\text {cyc }}$ (expected to be proportional to $\eta_{\mathrm{T}}$; solid line) and the microscopic magnetic diffusivity (dotted line).

pendence on $\operatorname{Re}_{M}$ is found to be weak which is also in accordance with the analytical studies. More recent nonlinear test-field calculations (Brandenburg et al. 2008b), indicate a similar dependence.

The present results may have implications for solar dynamo simulations. Clearly, the hope is that cyclic reversals occur on time scales that are asymptotically independent of the resistive time scale. What is less clear, however, is when asymptotic behavior sets in. Evidently, even for the highest $\operatorname{Re}_{M}$ simulations presented here the cycle frequency shows still a shallow decline with increasing magnetic Reynolds number; see the inset of Figure 11. On the other hand, the corresponding change in the turbulent magnetic diffusivity is only by a factor of a few, even when the magnetic Reynolds number changes by two orders of magnitude. This suggests that higher resolution simulations are needed to have any hope in seeing truly asymptotic behavior at large magnetic Reynolds numbers.

A modest level of quenching of $\eta_{\mathrm{t}}$ would certainly still be compatible with solar dynamo models. In fact, already since the early 1970s it was clear that standard solar dynamo models of $\alpha \Omega$ type show cycle frequencies that are too high for realistic parameters, so the models predicted cycle periods of about byears instead of 22 years (Köhler 1970). A modest quenching of $\eta_{\mathrm{t}}$ would therefore point in the right direction, but one would hope that the decrease in $\eta_{\mathrm{t}}$ would eventually level off. Unfortunately, this is not evident from any of the simulations presented so far.

As we have argued in the introduction, we do not expect the results for $\eta_{\mathrm{t}}\left(\operatorname{Re}_{M}\right)$ to depend on the presence or absence of magnetic helicity fluxes. However, this expectation should be verified using simulations. Allowing for such fluxes would mean that we have to abandon the assumption of periodic boundary conditions. Although open boundary conditions would imply saturation on a dynamical time scale (not the resistive one, as in the present case of periodic boundaries) the saturation amplitude would scale inversely with $\operatorname{Re}_{M}$, if it was not for the shear-mediated helicity fluxes that allow for large saturation amplitudes on a dynamical time scale (Brandenburg 2005, Käpylä et al. 2008).

The authors acknowledge the hospitality of NORDITA during the program "Turbulence and Dynamos." P. J. K. acknowledges financial support from the Finnish Academy grant No. 112431 and A. B. acknowledges support from the Swedish Research Council under grant No. 621-2007-4064. The numerical simulations were performed on the supercomputers hosted by CSC - IT Center for Science Ltd. in Espoo, Finland, who are financed by the Finnish ministry of education.

\section{REFERENCES}

Bhattacharjee, A., \& Yuan, Y. 1995, ApJ, 449, 739

Blackman, E.G., \& Brandenburg, A. 2002, ApJ, 579, 359 (BB02)

Blackman, E. G., \& Field, G. B. 2000, ApJ, 534, 984

Brandenburg, A. 2001, ApJ, 550, 824

Brandenburg, A. 2005, ApJ, 625, 539

Brandenburg, A., \& Käpylä, P.J., 2007, New Journal of Physics, 9, 305

Brandenburg, A., \& Subramanian, K. 2005a, A\&A, 439, 835

Brandenburg, A., \& Subramanian, K. 2005b, Astron. Nachr., 326, 400

Brandenburg, A., \& Subramanian, K. 2007, Astron. Nachr., 328, 507

Brandenburg, A., \& von Rekowski, B. 2001, A\&A, 379, 1153

Brandenburg, A., Bigazzi, A. \& Subramanian, K. 2001, MNRAS, 325, 685

Brandenburg, A., Dobler, W. \& Subramanian, K. 2002, AN, 323, 99

Brandenburg, A., Rädler, K.-H., Rheinhardt, M. \& Käpylä, P.J. 2008a, ApJ, 676, 740

Brandenburg, A., Rädler, K.-H., Rheinhardt, M. \& Subramanian, K. 2008b, ApJL, 687, L49

Cattaneo, F., \& Vainshtein, S. I. 1991, ApJ, 376, L21

Elperin, T., Kleeorin, N. \& Rogachevskii, I. 2003, PhRvE, 68, 016311

Field, G.B. \& Blackman, E. 2002, ApJ, 572, 685

Frisch, U., She, Z. S., \& Sulem, P. L. 1987, Physica D, 28, 382

Gruzinov, A. V., \& Diamond, P. H. 1994, Phys. Rev. Lett., 72, 1651

Gruzinov, A. V., \& Diamond, P. H. 1995, Phys. Plasmas, 2, 1941

Hughes, D. W., \& Proctor, M. R. E. 2009, PhRvL, 102, 044501

Käpylä, P.J., Korpi, M.J. \& Brandenburg, A. 2008, A\&A, 491, 353

Käpylä, P.J., Korpi, M.J. \& Brandenburg, A. 2009a, ApJ, submitted, arXiv:0812.3958
Käpylä, P.J., Mitra, D. \& Brandenburg, A. 2009b, PhRvE, 79, 016302

Kleeorin, N., Moss, D., Rogachevskii, I., \& Sokoloff, D. 2000, A\&A, 361, L5

Kitchatinov, L.L., Pipin, V.V. \& Rüdiger, G. 1994, AN, 315, 157

Köhler, H. 1970, Sol. Phys., 13, 3

Krause, F., \& Rädler, K.-H. 1980, Mean-field magnetohydrodynamics and dynamo theory (Oxford: Pergamon)

Maron, J., \& Blackman, E. G. 2002, ApJ, 566, L41

Moffatt, H. K. 1978, Magnetic field generation in electrically conducting fluids (Cambridge: Cambridge Univ. Press)

Mitra, D., Käpylä, P.J., Tavakol, R. \& Brandenburg, A. 2009, A\&A, in press, arXiv:0806.1608

Parker, E.N. 1979, Cosmical magnetic fields: Their origin and their activity (Oxford: Clarendon)

Rogachevskii, I. \& Kleeorin, N. 2001, PhRvE, 64, 056307

Stix, M. 1972, A\&A, 20, 9

Sur, S., Brandenburg, A. \& Subramanian, K. 2008, MNRAS, 385, L15

Tobias, S.M., Cattaneo, F. \& Brummell, N.H. 2008, ApJ, 685, 596

Vainshtein, S. I., \& Cattaneo, F. 1992, ApJ, 393, 165

Yousef, T.A., Brandenburg, A. \& Rüdiger, G. 2003, A\& A, 411, 321

Yousef, T.A., Heinemann, T., Schekochihin, A.A. et al. 2008a, PhRvL, 100, 184501

Yousef, T.A., Heinemann, T., Rincon, F. et al. 2008b, AN, 329, 737 